\title{
Beyond Interstitial Lung Disease: The Rapidly Evolving Role of Calgranulin B as a Biomarker of Systemic Malignancies
}

\author{
Shailendra Kapoor ${ }^{1,2,3}$ \\ KEY WORDS: calgranulin B; cholesteatomas; prostate cancer; ovarian carcinomas; prostate cancer.
}

\section{TO THE EDITOR}

The recent article by Bargagli et al. about the evaluation of Calgranulin B (S100A9) levels in the bronchoalveolar lavage fluid of patients with interstitial lung diseases was highly interesting and thought provoking [1]. Bargagli et al. have clearly shown that calgranulin B is involved in the evolution and progression of chronic lung diseases. Interestingly, calgranulin B is also rapidly evolving as a significant marker of a number of other systemic non-inflammatory conditions especially systemic malignances.

For instance, calgranulin B is increased in the stools of patients with colorectal cancer [2]. Similarly, Ott et al. have even demonstrated that fluid drawn from cystic malignant ovarian lesions express calgranulin B in sharp contrast to fluid drawn from benign cystic lesions which do not demonstrate calgranulin B [3]. Similarly, increased expression of calgranulin B is seen in biopsies of patients with Barretts esophagitis who demonstrate high grade dysplasia [4] while similar increased expression of calgranulin B is also seen in head and neck tumors such as cholesteatomas [5]. Increased expression of the calgranulin B gene has also been noted in pulmonary carcinomas in conjunction with increased expression of other genes such as apolipoprotein E [6]. Similarly, matrix-assisted laser desorption ionization-time of flight-mass spectroscopy (MALDI-TOF-MS) analysis of voided urine following prostatic massage from patients with prostate cancer reveals increased levels of calgranulin B/MRP-14 [7].

These examples clearly indicate the massive diagnostic as well as prognostic potential of calgranulin B. Clearly, there is a need for further large scale studies to elaborate the exact sensitivity and specificity of calgranulin B as a diagnostic marker in these various conditions so as to extend its use from beyond clinical studies to the realm of routine oncology.

\section{REFERENCES}

1. Bargagli, E., C. Olivieri, A. Prasse, et al. 2008. Calgranulin B (S100A9) levels in bronchoalveolar lavage fluid of patients with interstitial lung diseases. Inflammation. 31:351-354. doi:10.1007/ s10753-008-9085-z.

2. Yoo, B. C., Y. K. Shin, S. B. Lim, S. H. Hong, S. Y. Jeong, and J. G. Park. 2008. Evaluation of calgranulin B in stools from the patients with colorectal cancer. Dis. Colon Rectum. 51:1703-1709. doi:10.1007/s10350-008-9381-6.

3. Ott, H. W., H. Lindner, B. Sarg, et al. 2003. Calgranulins in cystic fluid and serum from patients with ovarian carcinomas 1. Cancer Res. 63:7507-7514.

4. Bax, D. A. 2007. High-grade dysplasia in Barrett's esophagus is associated with increased expression of calgranulin A and B. Scand. J. Gastroenterol. 42:902-910. doi:10.1080/00365520601138189.

5. Holly, A., J. Bujia, K. Lempart, and M. Serge. 1995. Expression of calgranulin A and B in middle ear cholesteatoma. Laryngorhinootologie. 74:33-35. doi:10.1055/s-2007-997682.

6. Zhang, H., B. Yue, Y. Jiyao, F. Qingtang, W. Yiming, and L. Neoplasms. 2007. Screening and identification of lung cancer associated proteins. J. Fourth Mil. Med. Univ. 28:6-8.

7. Rehman, I., A. R. Azzouzi, J. W. Catto, et al. 2004. Proteomic analysis of voided urine after prostatic massage from patients with prostate cancer: a pilot study. Urology. 64:1238-1243. doi:10.1016/ j.urology.2004.06.063.

\footnotetext{
${ }^{1}$ Schaumburg, IL 690195, USA

275 Kristin Circle \# 413, Schaumburg, IL 60195, USA

${ }^{3}$ To whom correspondence should be addressed at 75 Kristin Circle \# 413, Schaumburg, IL 60195, USA. E-mail: shailendrakapoor@yahoo.com
} 